\section{PILOT STUDY: THE VALIDITY OF THE QUEENS SQUARE SCREENING TEST FOR VISUAL DEFICITS IN A COHORT OF PATIENTS WITH DEMENTIA}

${ }^{1}$ Leah Kim*, ${ }^{2}$ Dennis Cordato, ${ }^{2}$ Alan McDougall, ${ }^{3}$ Clare Fraser. 'Sydney Eye Hospital, Sydney, NSW, Australia; ${ }^{2}$ Liverpool Hospital, Sydney, NSW, Australia; ${ }^{3}$ Save Sight Institute, Sydney, NSW, Australia

\subsection{6/jnnp-2019-anzan.114}

Introduction There is limited research on the visual deficits found in dementia. The Queens Square Screening Test for Visual Deficits (QS test) is designed to screen for changes in visual processing. Our study aimed to validate this test and examine the types of visual processing deficits found in dementia.

Methods We assessed the QS test in participants with dementia, mild cognitive impairment (MCI), and healthy controls. Participants were recruited from the Neurology and Geriatrics departments of a tertiary hospital over 3-months. Cognitive impairment was measured using the Rowland Universal Dementia Assessment Scale (RUDAS).

Results Twenty-six patients were examined. There were no statistically significant differences in age, gender, English fluency, and education between the three groups. Participants with dementia ( $n=8$, mean RUDAS 17.5/30) scored 51.4/71 on the QS test, compared to $60.7 / 71$ in MCI $(n=7$, mean RUDAS 25.0/30) and 64.6/71 in controls $(n=11$, mean RUDAS 27.4/30). The mean scores for each subset of the QS test for dementia, MCI and normal cognition, respectively, were: early visual processing - 19.6/25, 22.4/25, 23.7/25; object perception $-5.6 / 11,7.9 / 11,8.7 / 11$; space perception 11.4/14, 11.1/14, 12.4/14; face perception - 4.4/8, 6.4/8, 7.0/ 8 ; reading - 10.4/13, 12.9/13, 12.8/13.

Conclusion In this pilot study, the QS test was markedly abnormal in dementia but did not differentiate between MCI and normal cognition. Our findings suggest that deficits in early visual processing, reading, and the perception of objects and faces are common in dementia. Understanding the types of visual difficulties may improve the care of patients with dementia.

\section{CONTINUOUS EEG MONITORING IN AUSTRALIA: S FIRST YEAR AUDIT OF PRACTICE 2018-2019 OF A COMBINED HOSPITAL EPILEPSY PROGRAM IN MELBOURNE}

\footnotetext{
1,2,3 Joshua Laing* ${ }^{*}{ }^{1}$ Georgia Grant, ${ }^{1}$ Cecilia Harb, ${ }^{2}$ Elise White, ${ }^{1,2,3}$ Terence O'Brien, 1,2,3Patrick Kwan. 'Department of Neurology, Alfred Hospital, Melbourne, VIC, Australia; ${ }^{2}$ Department of Neurology, Royal Melbourne Hospital, Melbourne, VIC, Australia; ${ }^{3}$ Department of Neurosciences, Monash University, Melbourne, VIC, Australia
}

\subsection{6/jnnp-2019-anzan. 115}

Introduction Continuous EEG (cEEG) monitoring is essential for the optimal diagnosis and management of non-convulsive seizures. We audited the use of cEEG across two major metropolitan hospitals in Melbourne, Australia as part of a new combined epilepsy program. There are no reported case series of cEEG in Australia.

Methods Data was collected retrospectively from consecutive patients who underwent cEEG monitoring as part of their acute inpatient care between Jan 2018 to Dec 2018 at the Alfred and Royal Melbourne Hospitals. All inpatient EEG studies over $1 \mathrm{hr}$ were included. Elective cases from the epilepsy monitoring unit were excluded. Demographic and clinical information regarding their admission was collected. Descriptive statistics, and comparative analysis was performed.

Results There were 94 patients identified that underwent cEEG. 50\% were male and 50\% female, with an average age of 51.3 yrs. $45 \%$ were performed in the ICU, and $55 \%$ on the acute medical ward. The average duration of recording per patient was 86.2 hrs or 3.6 days. Of 89 of 94 available cEEG reports, seizures were seen in $55 \%$, the majority being non-convulsive. Interictal discharges were seen in 60\%. 19\% (13/67 of available records) were dead at the time of the audit.

Conclusion Non-convulsive seizures and non-convulsive status epilepticus is common and widely underrecognized without cEEG. We present 94 cases as part of our newly expanding cEEG program across two major Australian hospitals over a one year period, $55 \%$ with seizures which were predominantly non-convulsive. A prospective database will be designed for further quality improvement and future research.

\section{CLADIN: CLADRIBINE AND INNATE IMMUNE RESPONSES}

${ }^{1}$ Mastura Monif*, ${ }^{2}$ Shokoufeh Abdollahi, ${ }^{2}$ Jim Stankovich, ${ }^{3,4}$ Vicki Maltby, ${ }^{3,4}$ Jeannette Lechner-Scott, ${ }^{5,6}$ Tomas Kalincik, ${ }^{6} J o h n$ Hamilton, Terence O'Brien 2, 5, 7 Helmut Butzkueven 2, 7. 'Monash University, Melbourne Health, Alfred Health, Melbourne, VIC, Australia; ${ }^{2}$ Neurosciences, Monash University, Melbourne, VIC, Australia; ${ }^{3}$ Department of Neurology, John Hunter Hospital, New Lambton Heights, NSW, Australia; ${ }^{4}$ Hunter Medical Research Institute, School of Medicine and Public Health, and Centre for Brain and Mind Research, University of Newcastle, Callaghan, NSW, Australia; ${ }^{5}$ Department of Neurology, Royal Melbourne Hospital, Melbourne, VIC, Australia; ${ }^{6}$ Department of Medicine, The University of Melbourne, Melbourne, VIC, Australia; ${ }^{7}$ Department of Neurology, Alfred Health, Melbourne, VIC, Australia

\subsection{6/jnnp-2019-anzan. 116}

Introduction Cladribine Tablets $\left(\right.$ Mavenclad $\left.^{\circledR}\right)$ is nucleoside analogue of deoxyadenosine, and an oral treatment for relapsing remitting MS (RRMS). In RRMS clinical trials, Cladribine has been shown to reduce brain atrophy, relapse rates, and new lesions on brain MRI. P2X7R is a purinergic receptor expressed in innate immune cells, and is thought to play a critical role in neuroinflammation. The mechanism of action of Cladribine on peripheral innate immune cells (monocytes), and its effect on P2X7R, is unclear, and forms the basis of this study.

Methods This will be a Phase IV, multi-centre, 3 year, translational trial. Patients who are starting Cladribine as part of their routine clinical care will consent to take part in the study. Monocyte numbers and activation states will be measured at various times prior and after commencement of therapy. In addition, and in an in vitro setting the effect of Cladribine on P2X7R expression and function will be assessed, as well as measuring various cytokines/chemokines in serum. The laboratory data will also be correlated with clinical data from another long-term Cladribine study, CLOBAS.

Results This study has been approved by Alfred Health Human Research Ethics Committee. The study is to commence in April 2019.

Conclusion This study will shed light on whether Cladribine is exerting its beneficial effects via action on peripheral monocytes and alterations of their P2X7Rs. The laboratory and clinical data will be analysed to understand the relationship between innate immune parameters and patient outcome. 